FOLIA

Amazónica

Revista del Instituto de Investigaciones

de la Amazonía Peruana

\title{
DIVERSIDAD PLANCTÓNICA DE LOS CUERPOS DE AGUA EN LA RESERVA NACIONAL ALLPAHUAYO MISHANA, LORETO - PERÚ
}

\author{
Carlos CHÁVEZ-VEINTEMILLA ${ }^{1}$; Roberto PEZO $^{1}$; Elvis VASQUEZ ${ }^{2}$ \\ 1 Universidad Nacional de la Amazonía Peruana, Iquitos, Perú. \\ 2 Universidade do Estado do Amazonas - UEA, Manaus, Brasil. \\ * Correo electrónico: chavezcarlos72@hotmail.com
}

\section{RESUMEN}

El objetivo del presente estudio fue determinar la diversidad planctónica existente en los diferentes cuerpos de agua de la Reserva Nacional Allpahuayo Mishana (RNAM), Loreto Perú. La investigación se realizó en 16 estaciones ubicadas en cuerpos de agua lóticos y lénticos. La colecta, identificación y análisis de comunidades biológicas fue realizada empleando la metodología del MINAM \& UNMSM (2014) y se midieron los índices de Shannon - Weaver, Margaleff, Simpson y Pielou usando el programa PAST. Encontramos en la época húmeda 39 especies de fitoplancton cuyos taxa dominantes fueron Bacillariophyta, Charophyta, Chlorophyta y Euglenozoa, y en la época seca se identificaron 63 especies, cuyos taxa dominantes fueron Bacillariophyta, Charophyta, Chlorophyta y Cyanobacteria. Para el zooplancton en la época húmeda se identificaron 17 especies y en la época seca 24 especies y coincidentemente estuvieron dominadas por los taxones Rotatoria, Protozoa y Arthropoda. Los índices de Shannon - Weaver mostraron una biodiversidad media o normal; los de Margaleff que la diversidad estuvo relacionada con la riqueza y la abundancia de sus ambientes, los de Simpson que en general no hay dominio de una especie en particular, y los de Pielou que existen comunidades en equilibrio. Se concluye que las aguas de la cuenca media del río Nanay y la RNAM aún se conservan saludables, pese al impacto que se produce en su entorno.

PALABRAS CLAVE: Amazonía, área protegida, ecología, fitoplancton, zooplancton. 


\title{
PLANKTONIC DIVERSITY OF FRESH-WATER BODIES IN ALLPAHUAYO MISHANA NATIONAL RESERVE, LORETO - PERU
}

\begin{abstract}
The objective of this study was to determine the existing planktonic diversity in the different water bodies of the Allpahuayo Mishana National Reserve, Loreto Peru. The research was conducted in 16 stations located in lotic and lentic water bodies. The collection, identification and analysis of biological communities was carried out using the MINAM \& UNMSM (2014) methodology and the Shannon-Weaver, Margaleff, Simpson and Pielou indices were measured using the PAST program. We found 39 species of phytoplankton in the wet season, whose dominant taxa were Bacillariophyta, Charophyta, Chlorophyta and Euglenozoa, while in the dry season 63 species were identified, whose dominant taxa were Bacillariophyta, Charophyta, Chlorophyta and Cyanobacteria. For the zooplankton in the humid season, 17 species were identified and in the dry season 24 species and coincidentally they were dominated by the taxa Rotatoria, Protozoa and Arthropoda. The Shannon-Weaver indices showed an average or normal biodiversity; Margaleff that diversity was related to the richness and abundance of their environments, Simpson that in general there is no dominance of a particular species, and Pielou that there are communities in balance. We conclude that the waters of the middle basin of the Nanay River and Allpahuayo Mishana National Reserve are still healthy, despite the impact that occurs around it.
\end{abstract}

KEYWORDS: Amazonia, protected area, ecology, fitoplankton, zooplankton. 


\section{INTRODUCCIÓN}

La Reserva Nacional Allpahuayo Mishana - RNAM es una Área Nacional Protegida con 58,069.90 ha, y una zona de amortiguamiento equidistante de cincokilómetrosalrededor delamisma(SERNANP, 2013). El proyecto IIAP - BIODAMAZ (2004) logró identificar una serie de amenazas que ponen en riesgo su diversidad hidrobiológica, entre ellas, la destrucción de hábitats, la extracción selectiva y masiva de madera, la tala de algunas especies de árboles frutales, la extracción descontrolada de otros productos forestales, la extracción ilegal de material minero no metálico, la caza y la pesca sin control. Por ello, es imprescindible identificar la biota acuática para proponer planes de manejo adecuados, que contribuyan a la conservación y desarrollo sostenible de esta parte de la Amazonía peruana.

La RNAM comprende bosques a lo largo de la cuenca media del río Nanay que son inundados por sus aguas negras, donde habitan más de una docena de comunidades que subsisten de la comercialización a pequeña escala de sus recursos naturales, entre ellos los peces que son la base de su alimentación. El pescado es la principal fuente de proteína animal para el ser humano, y presenta a nivel mundial una demanda en continua expansión, debido al crecimiento de la población (IIAP (2009). Los recursos hidrobiológicos, son influenciados por fenómenos naturales, responsables por su presencia o ausencia en determinados períodos. De esta forma, Wittmann \& Junk (2016), indican que la fuerza responsable de la existencia, productividad e interacciones de la biota dominante en los sistemas río-planicie inundada es el pulso de inundación.

El pulso de inundación influencia en muchos aspectos a los organismos acuáticos, entre estos, al plancton, el cual está constituido por microorganismos que habitan los cuerpos de aguas que cumplen un papel importante en la cadena alimentaria debido a la transferencia de energía de un nivel trófico a otro (Lenz, 2000; Sipaúba-Tavares et al., 2001) y está compuesto por el fitoplancton y el zooplancton.

El fitoplancton es el ensamblaje de microorganismos, en su mayoría fotosintéticos, que viven suspendidos faltos de movimiento propio en la masa de agua (Cambra et al. 2005), los cuales pueden variar entre organismos unicelulares y pluricelulares, constituyendo los productores primarios en ambientes acuáticos continentales. El zooplancton es el grupo microscópico de animales flotantes con limitada locomoción, unicelulares o multicelulares, que van desde unos pocos micrómetros a un milímetro o más, y que además de las variaciones de tamaño, existen diferencias morfológicas y de posición taxonómica (Goswami, 2004).

Entre los estudios realizados en el área de influencia de la RNAM tenemos la migración pasiva del ictioplancton en la cuenca media del río Nanay (Ríos et al., 2002), la densidad y diversidad del fitoplancton de la cocha Llanchama, en los periodos hidrológicos de creciente y media vaciante, Loreto, Perú (López \& Vela, 2011), entre otros. El objetivo de este estudio es determinar la diversidad planctónica en la cuenca del río Nanay.

\section{MATERIALES Y MÉTODOS}

\section{ÁREA DE ESTUDIO}

Se consideraron los tipos de cuerpos de agua que existen en la cuenca del río Nanay, los lóticos (río Nanay, río Pintuyacu y las quebradas) y los lénticos (cochas o lagunas) monitoreados durante los períodos hidrológicos húmedo o lluvioso y seco. Se tuvieron dieciséis estaciones en total, cinco en el río Nanay (Pisco, Anguilla, Yarana, San Martín y Llanchama), uno en el río Pintuyacu con la estación del mismo nombre, cinco en quebradas 
(Curaca, Tocón, San Pedro, Santa Cruz y Agua Negra) y cinco en cochas (Samito, Lagunas, Yuto, Yarana y Supay Poza). (Figura 1 y Tabla 1 ).

Las características físicas y químicas del agua fueron medidas con kits para monitoreo de aguas y un multiparámetro Hanna YSI.

\section{EVALUACIÓN DEL FITOPLANCTON Y ZOOPLANCTON}

Para fitoplancton se colectaron tres muestras por cada punto de muestreo, filtrando 40 litros de agua por muestra con una red de 25 micras, el material filtrado fue colocado en frascos de polietileno de $250 \mathrm{ml}$, debidamente etiquetado (código de campo, fecha, colector) y fijado con formol al 5\% (MINAM \& UNMSM, 2014), posteriormente fueron identificadas con el uso de claves dicotómicas (Aldave, 1989; Bicudo \& Bicudo, 1969; Bicudo \& Menezes, 2006; Bourrelly
(1968, 1970, 1972); Fernández, 1982; Cadima \& Bicudo, 2014).

Para zooplancton se colectaron tres muestras por cada punto, filtrando 40 litros de agua por muestra con una red de 60 micras, el material filtrado fue colocado en frascos de polietileno de $250 \mathrm{ml}$, debidamente etiquetado (código de campo, fecha, colector) y fijado con formol al 5\% (MINAM \& UNMSM, 2014), luego fueron identificadas con el uso de claves dicotómicas (Eddy \& Hodson, 1961; Pennak, 1978).

\section{ANÁLISIS DE DATOS}

El análisis de datos se realizó con el software Excel versión 2013 para la elaboración de tablas y gráficos, y el programa estadístico PAST 3.17, para la obtención de los índices de diversidad. Se estimaron y compararon indicadores comunitarios de Riqueza específica absoluta (S),

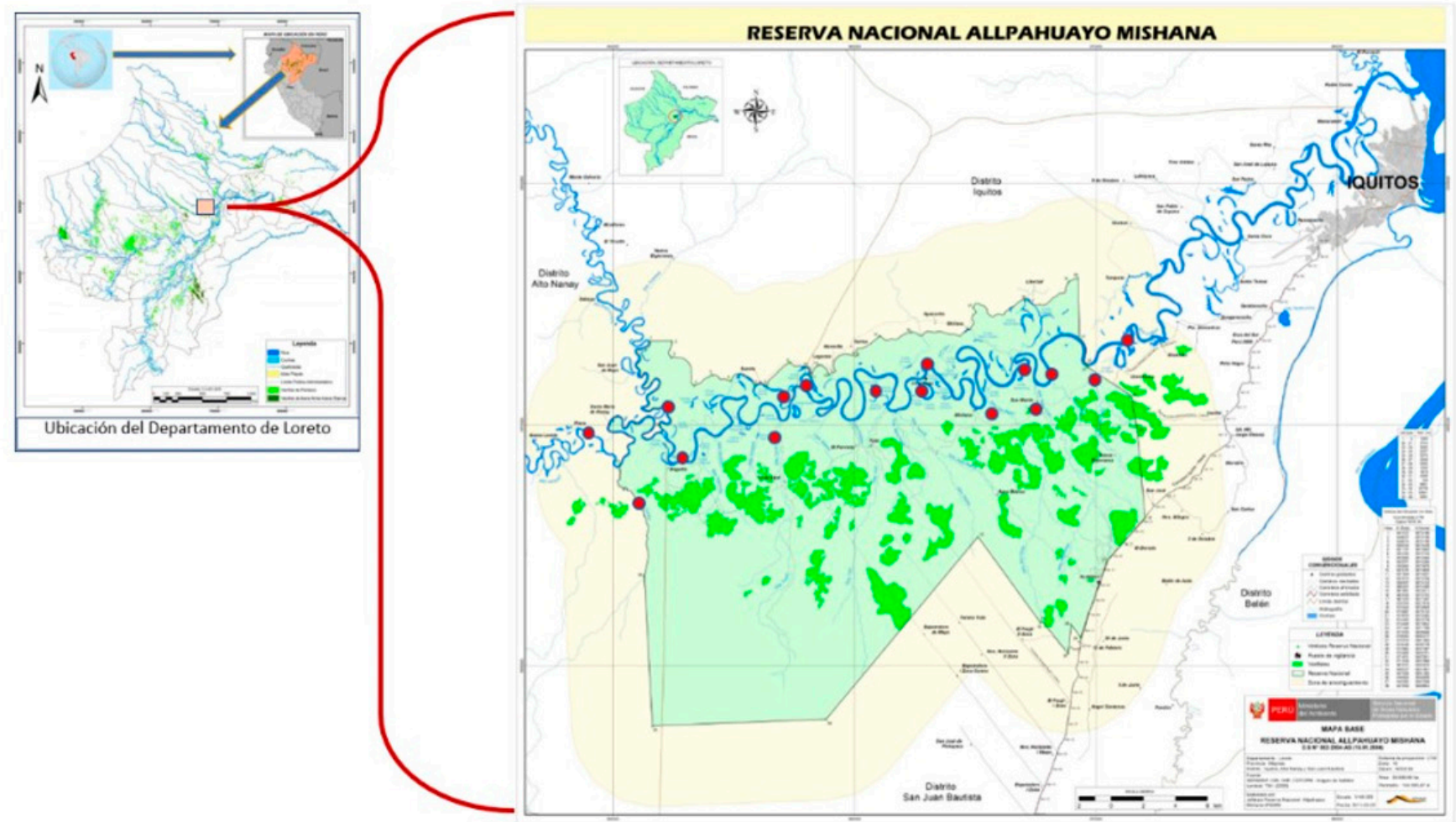

Figura 1. Ubicación de las estaciones de monitoreo en la cuenca del río Nanay, Reserva Nacional Allpahuayo Mishana (Loreto, Perú). 
Tabla 1: Estaciones de monitoreo de diversidad planctónica - Reserva Nacional Allpahuayo Mishana (Loreto, Perú).

\begin{tabular}{|c|c|c|c|c|c|}
\hline \multirow{3}{*}{ Ecosistema } & \multirow{3}{*}{$\begin{array}{c}\text { Cuerpo de } \\
\text { agua }\end{array}$} & \multirow{3}{*}{$\begin{array}{c}\mathrm{N}^{\circ} \text { de } \\
\text { Orden } \\
\text { Asignado }\end{array}$} & \multirow{3}{*}{ Denominación } & \multicolumn{2}{|c|}{ Coordenadas UTM } \\
\hline & & & & \multicolumn{2}{|c|}{ WGS 84, Zona $18 \mathrm{M}$} \\
\hline & & & & Este & Norte \\
\hline & & 1 & Pisco (aguas arriba) & 643097 & 9569641 \\
\hline & & 2 & Anguilla & 649378 & 9567682 \\
\hline & Río Nanay & 3 & Yarana & 664186 & 9572809 \\
\hline & & 4 & San Martín & 671929 & 9572462 \\
\hline & & 5 & LLanchama (aguas abajo) & 677140 & 9575069 \\
\hline \multirow[t]{8}{*}{ Lótico } & Río Pintuyacu & 6 & Pintuyacu & 648618 & 9570250 \\
\hline & & 7 & Curaca & 645498 & 9567143 \\
\hline & & 8 & Tocón & 655298 & 9570071 \\
\hline & Quebrada & 9 & San Pedro & 668342 & 9571507 \\
\hline & & 10 & Santa Cruz & 671188 & 9572372 \\
\hline & & 11 & Agua Negra & 674474 & 9573476 \\
\hline & & 12 & Samito & 655760 & 9571059 \\
\hline & & 13 & Lagunas & 657010 & 9572412 \\
\hline \multirow[t]{3}{*}{ Léntico } & Cocha & 14 & Yuto & 661433 & 9571977 \\
\hline & & 15 & Yarana & 664080 & 9572272 \\
\hline & & 16 & Supay Poza & 670647 & 9573351 \\
\hline
\end{tabular}

Riqueza relativa $(\% \mathrm{~S})$ y abundancia relativa $(\% \mathrm{~N})$ de organismos colectados, Índices de diversidad (Shannon-Weaver, Margalef y Simpson), e Índice de equidad (Pielou). Todos estos indicadores fueron calculados a nivel de estaciones de monitoreo y temporada.

\section{RESULTADOS}

\section{PARÁMETROS FÍSICOS Y QUÍMICOS}

Las Tablas 2 y 3 muestran los valores de los parámetros medidos en campo, en la época húmeda $\mathrm{y}$ seca, respectivamente, lo cual representa un ambiente de aguas negras.
COMPOSICIÓN, RIQUEZA, ABUNDANCIA Y DIVERSIDAD DEL FITOPLANCTON

En la época húmeda, se identificaron 39 especies correspondientes a 5 taxa, Bacillariophyta (56\%), Charophyta (21\%), Euglenozoa (10\%), Chlorophyta (8\%), y Myozoa (5\%) (Tabla 4 y Figura 2).

En setiembre del mismo año, en plena vaciante o época seca, se identificaron 63 especies, cuyos taxa fueron Bacillariophyta (59\%), Charophyta (19\%), Chlorophyta (8\%), Cyanobacteria (6\%), Euglenozoa (3\%), Myozoa, Cercizoa y Ochrophyta con $2 \%$, respectivamente (Tabla 4 y Figura 3). 
Tabla 2. Parámetros físicos y químicos de las estaciones de monitoreo en época húmeda en la cuenca del río Nanay, Reserva Nacional Allpahuayo Mishana (Loreto, Perú).

\begin{tabular}{|c|c|c|c|c|c|c|c|c|}
\hline \multirow[b]{2}{*}{ Ecosistema } & \multirow[b]{2}{*}{$\begin{array}{c}\text { Cuerpo de } \\
\text { agua }\end{array}$} & \multirow[b]{2}{*}{ Denominación } & \multicolumn{6}{|c|}{ Parámetros } \\
\hline & & & $\begin{array}{l}\text { Profundi- } \\
\text { dad (m) }\end{array}$ & $\begin{array}{l}\text { DO } \\
\text { (ppm) }\end{array}$ & $\mathrm{pH}$ & $\begin{array}{l}\text { Tempera- } \\
\text { tura Agua } \\
\left({ }^{\circ} \mathrm{C}\right)\end{array}$ & $\begin{array}{l}\text { Conducti- } \\
\text { vidad } \\
(\mu \mathrm{S} / \mathrm{cm})\end{array}$ & $\begin{array}{l}\text { Trans- } \\
\text { parencia } \\
(\mathrm{cm})\end{array}$ \\
\hline \multirow{11}{*}{ Lótico } & \multirow{5}{*}{$\begin{array}{l}\text { Río } \\
\text { Nanay }\end{array}$} & Pisco (aguas arriba) & 9,0 & 2,2 & 4,9 & 25,8 & 10,0 & 150,0 \\
\hline & & Anguilla & 9,5 & 2,6 & 4,9 & 25,9 & 9,8 & 110,0 \\
\hline & & Yarana & 11,0 & 2,8 & 4,8 & 26,0 & 9,8 & 130,0 \\
\hline & & San Martín & 10,0 & 2,7 & 4,8 & 26,3 & 9,7 & 120,0 \\
\hline & & $\begin{array}{l}\text { LLanchama } \\
\text { (aguas abajo) }\end{array}$ & 9,0 & 2,5 & 4,9 & 26,3 & 9,0 & 120,0 \\
\hline & $\begin{array}{c}\text { Río } \\
\text { Pintuyacu }\end{array}$ & Pintuyacu & 10,0 & 3,7 & 5,0 & 26,0 & 10,3 & 70,0 \\
\hline & \multirow{5}{*}{ Quebrada } & Curaca & 6,5 & 3,5 & 4,8 & 24,9 & 8,5 & 90,0 \\
\hline & & Tocón & 7,0 & 1,2 & 4,7 & 25,5 & 8,7 & 110,0 \\
\hline & & San Pedro & 6,0 & 2,6 & 4,8 & 26,1 & 9,0 & 110,0 \\
\hline & & Santa Cruz & 7,0 & 1,5 & 4,9 & 26,1 & 8,8 & 120,0 \\
\hline & & Agua Negra & 6,0 & 1,3 & 5,0 & 26,2 & 9,0 & 120,0 \\
\hline \multirow{5}{*}{ Léntico } & \multirow{5}{*}{ Cocha } & Samito & 6,0 & 2,4 & 4,9 & 26,0 & 8,0 & 100,0 \\
\hline & & Lagunas & 7,0 & 2,8 & 4,8 & 26,0 & 8,5 & 100,0 \\
\hline & & Yuto & 7,0 & 2,4 & 5,0 & 26,4 & 7,8 & 120,0 \\
\hline & & Yarana & 7,0 & 2,6 & 4,9 & 26,1 & 7,7 & 110,0 \\
\hline & & Supay Poza & 7,0 & 2,5 & 4,9 & 26,7 & 9,0 & 120,0 \\
\hline
\end{tabular}


Tabla 3: Parámetros físicos y químicos de las estaciones de monitoreo en época seca en la cuenca del río Nanay, Reserva Nacional Allpahuayo Mishana (Loreto, Perú)..

\begin{tabular}{|c|c|c|c|c|c|c|c|c|}
\hline \multirow[b]{2}{*}{ Ecosistema } & \multirow[b]{2}{*}{$\begin{array}{l}\text { Cuerpo de } \\
\text { agua }\end{array}$} & \multirow[b]{2}{*}{ Denominación } & \multicolumn{6}{|c|}{ Parámetros } \\
\hline & & & $\begin{array}{l}\text { Profundi- } \\
\text { dad (m) }\end{array}$ & $\begin{array}{l}\text { DO } \\
\text { (ppm) }\end{array}$ & $\mathrm{pH}$ & $\begin{array}{c}\text { Tempera- } \\
\text { tura Agua } \\
\left({ }^{\circ} \mathrm{C}\right)\end{array}$ & $\begin{array}{l}\text { Conduc- } \\
\text { tividad } \\
(\mu \mathrm{S} / \mathrm{cm})\end{array}$ & $\begin{array}{l}\text { Trans- } \\
\text { parencia } \\
\text { (cm) }\end{array}$ \\
\hline \multirow{11}{*}{ Lótico } & \multirow{5}{*}{$\begin{array}{l}\text { Río } \\
\text { Nanay }\end{array}$} & Pisco (aguas arriba) & 2,0 & 6,3 & 5,0 & 27,9 & 9,5 & 70,0 \\
\hline & & Anguilla & 1,0 & 6,0 & 4,3 & 27,9 & 8,0 & 70,0 \\
\hline & & Yarana & 1,5 & 5,8 & 4,0 & 28,3 & 8,2 & 75,0 \\
\hline & & San Martín & 2,0 & 5,3 & 4,4 & 27,1 & 8,5 & 70,0 \\
\hline & & $\begin{array}{l}\text { LLanchama } \\
\text { (aguas abajo) }\end{array}$ & 4,5 & 5,5 & 4,1 & 29,3 & 8,1 & 65,0 \\
\hline & $\begin{array}{c}\text { Río } \\
\text { Pintuyacu }\end{array}$ & Pintuyacu & 3,3 & 5,8 & 4,4 & 27,5 & 8,0 & 65,0 \\
\hline & \multirow{5}{*}{ Quebrada } & Curaca & 2,0 & 6,3 & 4,5 & 25,8 & 8,0 & 65,0 \\
\hline & & Tocón & 1,5 & 6,5 & 4,3 & 26,2 & 8,2 & 60,0 \\
\hline & & San Pedro & 1,0 & 5,4 & 4,4 & 26,1 & 8,5 & 85,0 \\
\hline & & Santa Cruz & 1,5 & 5,9 & 5,2 & 29,2 & 8,5 & 65,0 \\
\hline & & Agua Negra & 1,5 & 5,9 & 4,6 & 27,6 & 8,8 & 70,0 \\
\hline \multirow{5}{*}{ Léntico } & \multirow{5}{*}{ Cocha } & Samito & 3,0 & 5,5 & 4,2 & 30,8 & 7,9 & 105,0 \\
\hline & & Lagunas & 3,5 & 4,7 & 4,2 & 30,1 & 8,2 & 110,0 \\
\hline & & Yuto & 4,0 & 4,9 & 4,2 & 29,7 & 7,7 & 100,0 \\
\hline & & Yarana & 3,0 & 4,9 & 4,4 & 30,8 & 7,8 & 110,0 \\
\hline & & Supay Poza & 2,0 & 6,5 & 4,2 & 29,8 & 8,4 & 80,0 \\
\hline
\end{tabular}


Tabla 4. Composición y Riqueza del Fitoplancton y Zooplancton en la cuenca del río Nanay, Reserva Nacional Allpahuayo Mishana (Loreto, Perú).

\begin{tabular}{|c|c|c|c|c|c|c|c|c|c|c|c|c|c|c|c|c|c|c|c|c|c|c|c|c|c|c|c|c|c|c|c|c|c|c|}
\hline \multirow{2}{*}{$\begin{array}{l}\text { Cuerpo de Agua } \\
\text { Estaciones }\end{array}$} & \multicolumn{12}{|c|}{ Río } & \multicolumn{10}{|c|}{ Quebrada } & \multicolumn{10}{|c|}{ Cocha } & \multicolumn{2}{|c|}{$\begin{array}{c}\text { Total } \\
\text { Especies }\end{array}$} \\
\hline & \multicolumn{2}{|c|}{ Pisco } & \multicolumn{2}{|c|}{ Anguilla } & \multicolumn{2}{|c|}{ Yarana } & \multicolumn{2}{|c|}{ S. Martín } & \multicolumn{2}{|c|}{ Llanchama } & \multicolumn{2}{|c|}{ Pintuyacu } & \multicolumn{2}{|c|}{ Curaca } & \multicolumn{2}{|c|}{ Tocón } & \multicolumn{2}{|c|}{ S. Pedro } & \multicolumn{2}{|c|}{ S. Cruz } & \multicolumn{2}{|c|}{ A. Negra } & \multicolumn{2}{|c|}{ Samito } & \multicolumn{2}{|c|}{ Lagunas } & \multicolumn{2}{|c|}{ Yuto } & \multicolumn{2}{|c|}{ Yarana } & \multicolumn{2}{|c|}{ S. Poza } & & \\
\hline $\begin{array}{l}\text { Coordenadas } \\
\text { (Este/Norte) }\end{array}$ & $\begin{array}{l}064 \\
956\end{array}$ & 3097 & & $\begin{array}{l}378 \\
682\end{array}$ & $\begin{array}{l}066 \\
957\end{array}$ & $\begin{array}{l}1186 \\
809\end{array}$ & $\begin{array}{l}067 \\
957\end{array}$ & $\begin{array}{l}929 \\
462\end{array}$ & & 140 & $\begin{array}{l}064 \\
957\end{array}$ & $\begin{array}{l}3618 \\
250\end{array}$ & $\begin{array}{l}064 \\
956\end{array}$ & $\begin{array}{l}5498 \\
7143\end{array}$ & $\begin{array}{l}0655 \\
9570\end{array}$ & 298 & $\begin{array}{l}066 \\
957\end{array}$ & $\begin{array}{l}3342 \\
507\end{array}$ & & 2378 & & 4474 & $\begin{array}{l}065 \\
957\end{array}$ & $\begin{array}{l}5760 \\
059\end{array}$ & & 010 & $\begin{array}{l}066 \\
957\end{array}$ & $\begin{array}{l}1433 \\
977\end{array}$ & $\begin{array}{l}066 \\
957\end{array}$ & $\begin{array}{l}4080 \\
2272\end{array}$ & $\begin{array}{l}067 \\
957\end{array}$ & $\begin{array}{l}0647 \\
3351\end{array}$ & & \\
\hline Phylum \Época & ES & $\mathrm{EH}$ & ES & $\mathrm{EH}$ & ES & $\mathrm{EH}$ & ES & $\mathrm{EH}$ & ES & $\mathrm{EH}$ & ES & $\mathrm{EH}$ & ES & $\mathrm{EH}$ & ES & $\mathrm{EH}$ & ES & $\mathrm{EH}$ & ES & $\mathrm{EH}$ & ES & $\mathrm{EH}$ & ES & $\mathrm{EH}$ & ES & $\mathrm{EH}$ & ES & $\mathrm{EH}$ & ES & $\mathrm{EH}$ & ES & $\mathrm{EH}$ & ES & $\mathrm{EH}$ \\
\hline Euglenozoa & 0 & 0 & 0 & 0 & 0 & 0 & 0 & 1 & 0 & 2 & 0 & 0 & 0 & 0 & 0 & 1 & 0 & 1 & 1 & 0 & 0 & 0 & 0 & 0 & 0 & 0 & 0 & 1 & 0 & 0 & 1 & 0 & 2 & 4 \\
\hline Bacillariophyta & 13 & 2 & 6 & 4 & 2 & 0 & 3 & 3 & 7 & 1 & 2 & 5 & 4 & 2 & 7 & 5 & 5 & 4 & 5 & 4 & 9 & 1 & 0 & 4 & 2 & 2 & 2 & 3 & 1 & 2 & 7 & 0 & 37 & 22 \\
\hline Chlorophyta & 0 & 0 & 0 & 0 & 0 & 0 & 1 & 0 & 2 & 1 & 2 & 0 & 0 & 1 & 0 & 0 & 2 & 1 & 0 & 0 & 0 & 0 & 1 & 1 & 0 & 0 & 1 & 0 & 1 & 0 & 1 & 0 & 5 & 3 \\
\hline Charophyta & 0 & 0 & 2 & 1 & 4 & 2 & 1 & 0 & 0 & 1 & 1 & 0 & 1 & 3 & 1 & 0 & 2 & 4 & 2 & 1 & 1 & 3 & 2 & 1 & 3 & 0 & 3 & 0 & 4 & 3 & 1 & 1 & 12 & 8 \\
\hline Miozoa & 0 & 0 & 0 & 1 & 0 & 0 & 0 & 1 & 0 & 0 & 0 & 0 & 1 & 1 & 0 & 0 & 0 & 0 & 0 & 0 & 0 & 0 & 0 & 0 & 0 & 0 & 0 & 0 & 0 & 0 & 1 & 0 & 1 & 2 \\
\hline Cyanobacteria & 0 & 0 & 1 & 0 & 2 & 0 & 0 & 0 & 3 & 0 & 3 & 0 & 0 & 0 & 0 & 0 & 0 & 0 & 0 & 0 & 1 & 0 & 3 & 0 & 1 & 0 & 0 & 0 & 0 & 0 & 0 & 0 & 4 & 0 \\
\hline Ochrophyta & 0 & 0 & 0 & 0 & 1 & 0 & 0 & 0 & 0 & 0 & 1 & 0 & 0 & 0 & 0 & 0 & 0 & 0 & 0 & 0 & 0 & 0 & 0 & 0 & 0 & 0 & 0 & 0 & 0 & 0 & 0 & 0 & 1 & 0 \\
\hline Cercizoa & 0 & 0 & 0 & 0 & 1 & 0 & 1 & 0 & 0 & 0 & 0 & 0 & 0 & 0 & 0 & 0 & 0 & 0 & 0 & 0 & 0 & 0 & 0 & 0 & 0 & 0 & 0 & 0 & 0 & 0 & 0 & 0 & 1 & 0 \\
\hline Total Fitoplancton & 13 & 2 & 9 & 6 & 10 & 2 & 6 & 5 & 12 & 5 & 9 & 5 & 6 & 7 & 8 & 6 & 9 & 10 & 8 & 5 & 11 & 4 & 6 & 6 & 6 & 2 & 6 & 4 & 6 & 5 & 11 & 1 & 63 & 39 \\
\hline Protozoa & 0 & 2 & 2 & 2 & 1 & 2 & 1 & 4 & 1 & 2 & 0 & 3 & 1 & 3 & 1 & 2 & 2 & 1 & 2 & 2 & 1 & 2 & 0 & 2 & 0 & 1 & 0 & 2 & 1 & 3 & 0 & 3 & 5 & 6 \\
\hline Rotatoria & 2 & 0 & 1 & 2 & 4 & 1 & 0 & 2 & 5 & 3 & 2 & 0 & 0 & 1 & 2 & 3 & 4 & 0 & 4 & 1 & 3 & 3 & 3 & 2 & 2 & 1 & 3 & 3 & 8 & 2 & 3 & 4 & 17 & 7 \\
\hline Arthropoda & 0 & 0 & 0 & 2 & 1 & 0 & 1 & 0 & 1 & 2 & 0 & 3 & 0 & 0 & 1 & 0 & 0 & 1 & 0 & 0 & 0 & 0 & 0 & 0 & 1 & 1 & 0 & 1 & 2 & 0 & 1 & 0 & 2 & 4 \\
\hline Total Zooplancton & 2 & 2 & 3 & 6 & 6 & 3 & 2 & 6 & 7 & 7 & 2 & 6 & 1 & 4 & 4 & 5 & 6 & 2 & 6 & 3 & 4 & 5 & 3 & 4 & 3 & 3 & 3 & 6 & 11 & 5 & 4 & 7 & 24 & 17 \\
\hline Totales Plancton & 15 & 4 & 12 & 12 & 16 & 5 & 8 & 11 & 19 & 12 & 11 & 11 & 7 & 11 & 12 & 11 & 15 & 12 & 14 & 8 & 15 & 9 & 9 & 10 & 9 & 5 & 9 & 10 & 17 & 10 & 15 & 8 & 87 & 56 \\
\hline
\end{tabular}




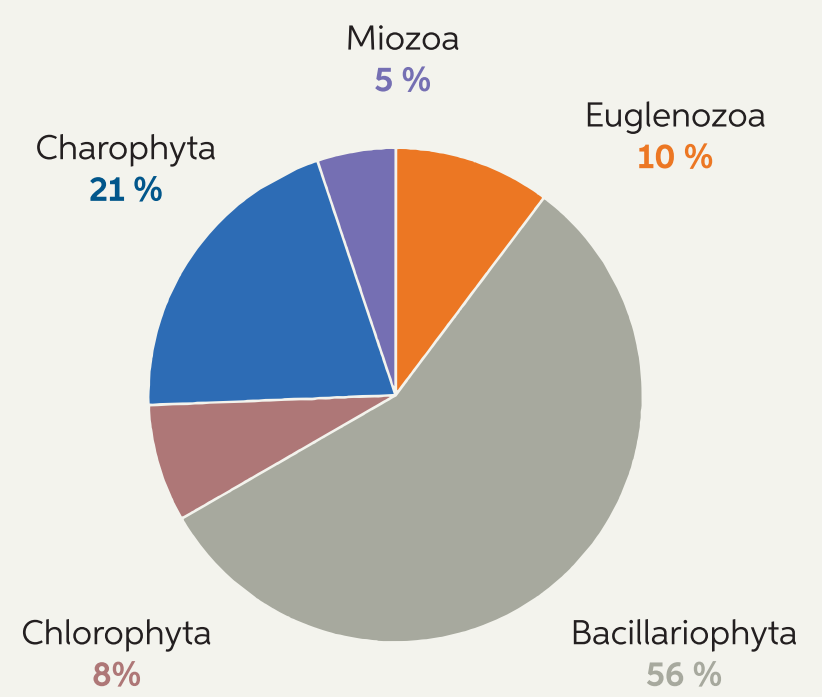

Figura 2. Composición del fitoplancton en época húmeda en la cuenca del río Nanay, Reserva Nacional Allpahuayo Mishana (Loreto, Perú).

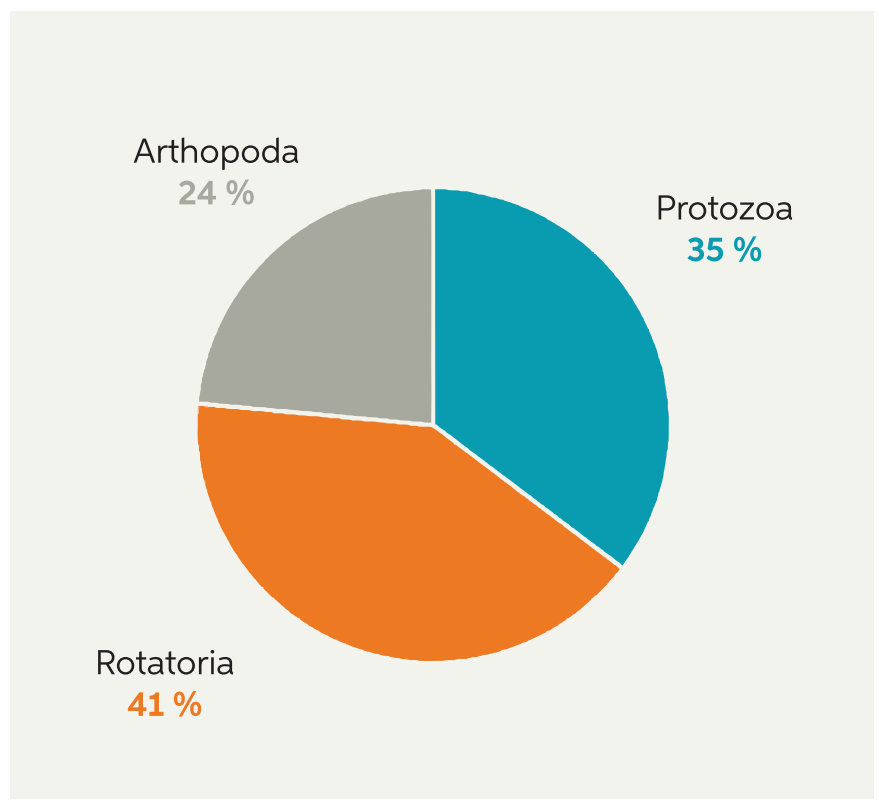

Figura 4. Composición del zooplancton en época seca en la cuenca del río Nanay, Reserva Nacional Allpahuayo Mishana (Loreto, Perú).

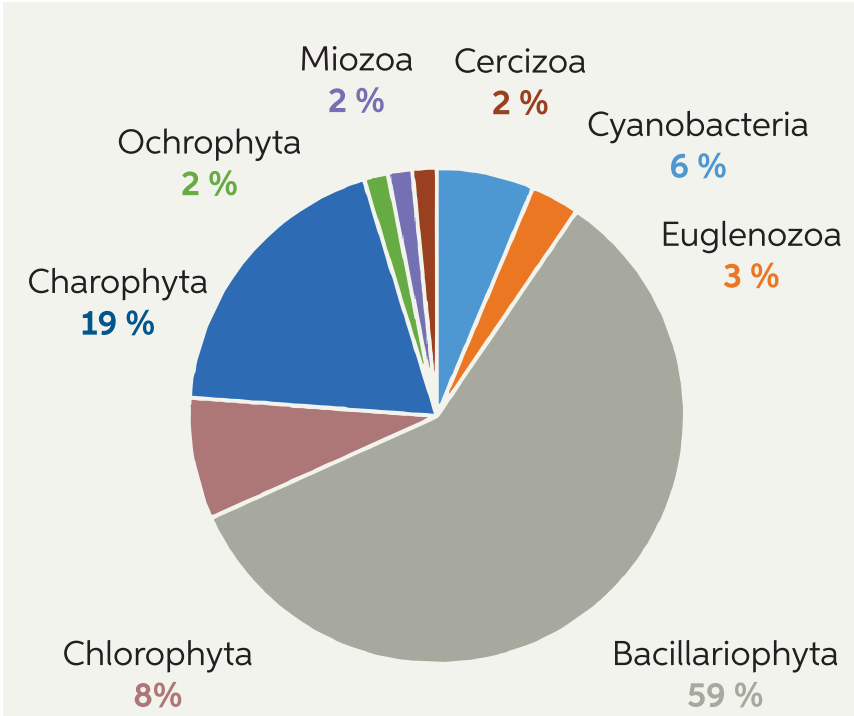

Figura 3. Composición del fitoplancton en época seca en la cuenca del río Nanay, Reserva Nacional Allpahuayo Mishana (Loreto, Perú).

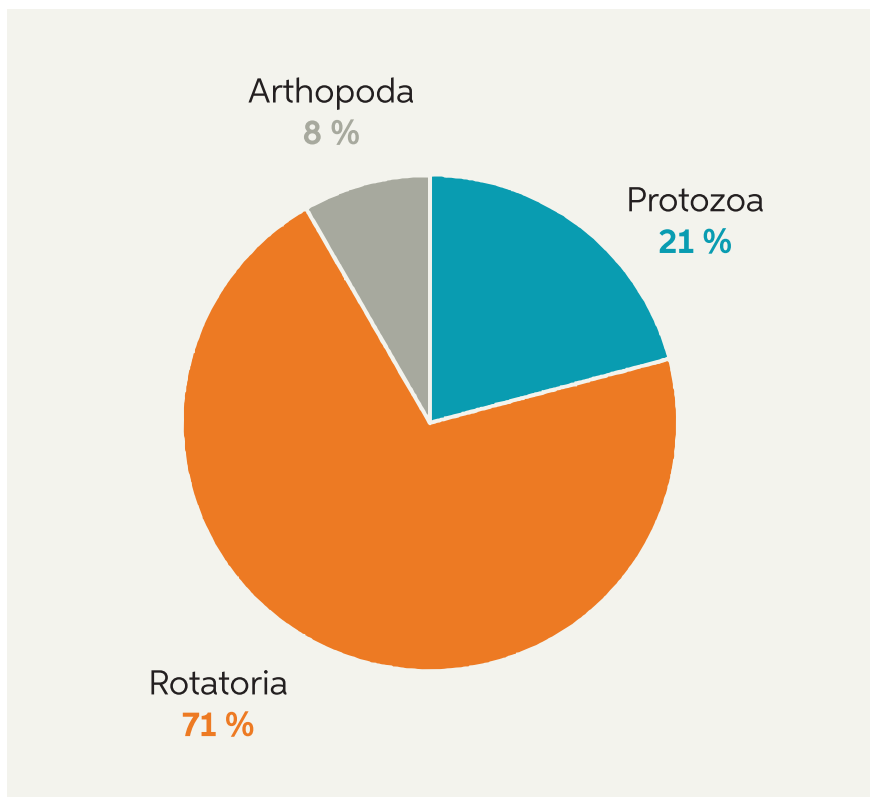

Figura 5. Composición del zooplancton en época húmeda en la cuenca del río Nanay, Reserva Nacional Allpahuayo Mishana (Loreto, Perú). 
De las 16 estaciones estudiadas, 13 de ellas $(81,25 \%)$ presentaron mayor riqueza en la época seca comparada con la época húmeda, dos estaciones de quebradas $(12,5 \%)$ presentaron menor riqueza en la época seca comparada con la época húmeda, y una estación de cocha $(6,25 \%)$ presentó similar riqueza en ambas épocas (Tabla 4).

Según el número de especies, de las estaciones ubicadas en el cuerpo de agua principal (río Nanay), las de mayor riqueza fueron Pisco (13 especies), LLanchama (12) y Yarana (10). A nivel de quebradas, Agua Negra (11), San Pedro (9), luego Tocón y Santa Cruz con 8 especies cada una. A nivel de cochas Supay Poza (11), seguida de Samito, Lagunas, Yuto y Yarana con 6 especies cada una.

En la época seca se registraron 350 individuos, mientras que en la época húmeda se registraron 150 individuos haciendo un total de 500 individuos. A nivel de taxa, Bacillariophyta fue el más abundante (61\%), seguido de Charophyta $(19,6 \%)$, Chlorophyta $(8,4 \%)$, Cyanobacteria (6\%), Euglenozoa (2,2\%), Miozoa (1,2\%), Ochrophyta ( $1 \%$ ) y Cercizoa (0,6 \%) (Tabla 5 ).

Los índices de diversidad se aprecian en la Tabla 6, donde los índices más altos de ShannonWeaver se registraron en la quebrada Agua Negra y en las estaciones de río (Pisco, Yarana y Llanchama), todas ellas en temporada seca, mientras que, en temporada húmeda, la quebrada San Pedro obtuvo un índice de $\mathrm{H}=2,30$ bits; considerándose estos valores como de diversidad normal.

A nivel de medias y según los cuerpos de agua, en la época seca la mayor diversidad estuvo en las estaciones ubicadas en el cauce principal de los ríos, luego las quebradas y las cochas. Entretanto, en la época húmeda, el orden según las medias fue quebradas, ríos y cochas.

El índice de Margalef más alto se registró en la estación Pisco, seguido de las quebradas Agua Negra y Tocón, todas en la temporada seca. A nivel de medias por cuerpos de agua el orden fue ríos, quebradas y cochas. Mientras que en la temporada húmeda el índice de Margalef más alto fue registrado en quebrada San Pedro, y a nivel de medias por cuerpos de agua fue mayor en las quebradas, ríos y cochas.

En la estación seca, los valores más altos para el índice de Simpson se registraron en las cochas Yuto $(D=0,9)$, Samito y Yarana ( $D=0,24$ cada uno). Estos valores nos indican que no existe dominio de una especie en particular, es decir, existe una baja dominancia. El orden según medias por tipo de cuerpos de agua fue cochas, ríos y quebradas. En la época húmeda cocha Supay Poza presentó una sola especie y la más alta dominancia $(D=1,00)$; mientras que la estación con menor dominancia fue la quebrada San Pedro $(D=0,10)$. A nivel de medias los mayores índices estuvieron en las cochas, seguido de los ríos y por último las quebradas.

Los índices de equidad en la época seca fueron superiores a 0,84. La estación con mayor equidad fue observada en la quebrada Tocón $(J=0,98)$, mientras que a nivel de medias y cuerpos de agua el orden fue quebradas, ríos y cochas. En época húmeda, la quebrada San Pedro obtuvo un índice de 1,00, al presentar 10 especies con un individuo cada una. El orden según las medias por cuerpo de agua fue quebradas, cochas y estaciones en río. Dichos valores demuestran una equidad relativamente alta y una distribución relativamente homogénea.

\section{COMPOSICIÓN, RIQUEZA, ABUNDANCIA Y DIVERSIDAD DEL ZOOPLANCTON}

En la época húmeda se identificaron 17 especies correspondientes a Rotatoria (41\%), Protozoa (35\%) y Arthropoda (24\%); mientras que en la época seca se identificaron 24 especies en la misma secuencia Rotatoria (71\%), Protozoa (21\%) y Arthropoda (8\%) (Tabla 4 y Figuras 4 y 5). 
Tabla 5. Abundancia de fitoplancton en la cuenca del río Nanay, Reserva Nacional Allpahuayo Mishana (Loreto, Perú).

\begin{tabular}{|c|c|c|c|c|c|}
\hline \multicolumn{3}{|c|}{ Fitoplancton } & \multirow{2}{*}{ Época Seca } & \multirow{2}{*}{ Época Húmeda } & \multirow{2}{*}{ TOTAL } \\
\hline Phylum & Familia & Especie & & & \\
\hline \multirow{4}{*}{ Cyanobacteria } & \multirow{3}{*}{ Oscillatoriaceae } & Oscillatoria sp. & 11 & 0 & 11 \\
\hline & & Oscillatoria formosa & 8 & 0 & 8 \\
\hline & & Oscillatoria limnetica & 10 & 0 & 10 \\
\hline & Spirulinaceae & Spirulina abbreviata & 1 & 0 & 1 \\
\hline \multirow{5}{*}{ Euglenozoa } & Euglenaceae & Trachelomonas armata & 1 & 0 & 1 \\
\hline & Phacaceae & Phacus sp. & 0 & 2 & 2 \\
\hline & Phacaceae & Phacus curvicauda & 0 & 1 & 1 \\
\hline & Phacaceae & Euglena sp. & 0 & 2 & 2 \\
\hline & Euglenaceae & Trachelomonas volvocina & 1 & 4 & 5 \\
\hline \multirow{23}{*}{ Bacillariophyta } & \multirow{4}{*}{ Fragilariaceae } & Fragilaria capucina & 5 & 2 & 7 \\
\hline & & Fragilaria construens & 3 & 0 & 3 \\
\hline & & Ceratoneis arcus & 2 & 6 & 8 \\
\hline & & Centronella sp. & 2 & 3 & 5 \\
\hline & Licmophoraceae & Licmophora gracilis & 7 & 1 & 8 \\
\hline & \multirow{3}{*}{ Tabellariaceae } & Tabellaria floculosa & 5 & 16 & 21 \\
\hline & & Diatoma vulgare & 0 & 1 & 1 \\
\hline & & Tabellaria binalis & 1 & 3 & 4 \\
\hline & \multirow{5}{*}{ Eunotiaceae } & Eunotia sp. & 8 & 3 & 11 \\
\hline & & Eunotia lunaris & 1 & 0 & 1 \\
\hline & & Actinella brasiliensis & 2 & 5 & 7 \\
\hline & & Actinella mirabilis & 4 & 0 & 4 \\
\hline & & Amphicampa sp. & 1 & 2 & 3 \\
\hline & & Navicula sp. & 20 & 13 & 33 \\
\hline & & Navicula dicephala & 7 & 1 & 8 \\
\hline & & Navicula espirata & 2 & 4 & 6 \\
\hline & & Navicula cryptocephala & 3 & 8 & 11 \\
\hline & Naviculaceae & Navicula peregrina & 2 & 0 & 2 \\
\hline & & Navicula simplex & 2 & 0 & 2 \\
\hline & & Gyrosigma sp. & 21 & 0 & 21 \\
\hline & & Gyrosigma acuminatum & 11 & 0 & 11 \\
\hline & & Stichococcus sp. & 2 & 0 & 2 \\
\hline & \multirow{3}{*}{ Pinnulariaceae } & Pinnularia sp. & 21 & 10 & 31 \\
\hline \multirow[t]{2}{*}{$\ldots$} & & Pinnularia biceps & 3 & 0 & 3 \\
\hline & & Diatomella sp. & 2 & 0 & 2 \\
\hline
\end{tabular}




\begin{tabular}{|c|c|c|c|c|c|}
\hline & & Nitzschia sp. & 3 & 1 & 4 \\
\hline \multirow{12}{*}{ Bacillariophyta } & \multirow[t]{2}{*}{ Bacillariaceae } & Nitzschia amphibia & 1 & 0 & 1 \\
\hline & & Denticula subtilis & 4 & 0 & 4 \\
\hline & Cymbellaceae & Cymbella sp. & 2 & 1 & 3 \\
\hline & Rhopalodiaceae & Epithemia sp. & 2 & 1 & 3 \\
\hline & \multirow{2}{*}{ Surirellaceae } & Surirella linearis & 14 & 1 & 15 \\
\hline & & Surirella biseriata & 1 & 0 & 1 \\
\hline & Rhoiscopeniaceae & Rhoicosphenia curvata & 4 & 3 & 7 \\
\hline & Aulacoseiraceae & Aulacoseira granulata & 34 & 0 & 34 \\
\hline & Amphipleuraceae & Frustulia rhomboides & 2 & 0 & 2 \\
\hline & Melosiraceae & Melosira sp. & 11 & 2 & 13 \\
\hline & Rhizosoleniaceae & Urosolenia sp. & 1 & 0 & 1 \\
\hline & Leptocylindraceae & Leptocylindrus minimus & 1 & 1 & 2 \\
\hline \multirow{5}{*}{ Chlorophyta } & Treubariaceae & Treubaria sp. & 2 & 1 & 3 \\
\hline & Chlorellaceae & Actinastrum gracilinum & 5 & 8 & 13 \\
\hline & Trebouxiophyceae & Crucigenia rectangulare & 8 & 0 & 8 \\
\hline & Ulotrichaceae & Ulotrix zonata & 14 & 1 & 15 \\
\hline & Chlorellaceae & Dictyophaerium sp. & 3 & 0 & 3 \\
\hline \multirow{15}{*}{ Charophyta } & \multirow{8}{*}{ Desmidiaceae } & Desmidium sp. & 2 & 0 & 2 \\
\hline & & Cosmarium sp. & 0 & 1 & 1 \\
\hline & & Triploceras sp. & 2 & 0 & 2 \\
\hline & & Groembladia neglecta & 2 & 0 & 2 \\
\hline & & Hyalotheca dissiliens & 14 & 6 & 20 \\
\hline & & Hyalotheca mucosa & 12 & 1 & 13 \\
\hline & & Hyalotheca undulata & 12 & 0 & 12 \\
\hline & & Euastrum laticeps & 0 & 2 & 2 \\
\hline & \multirow{3}{*}{ Closteriaceae } & Closterium acuiculare & 1 & 7 & 8 \\
\hline & & Closterium lunula & 2 & 0 & 2 \\
\hline & & Closterium moniliferum & 2 & 0 & 2 \\
\hline & \multirow{2}{*}{ Gonatozygoceae } & Gonatozygon kinahani & 2 & 6 & 8 \\
\hline & & Gonatozygon brebissoni & 2 & 0 & 2 \\
\hline & \multirow{2}{*}{ Zygnemataceae } & Mougeotia sp. & 5 & 4 & 9 \\
\hline & & Spirogyra sp. & 0 & 13 & 13 \\
\hline Ochrophyta & Tribonemaceae & Bumilleria sp. & 5 & 0 & 5 \\
\hline \multirow{2}{*}{ Miozoa } & Peridiniaceae & Protoperidinium sp. & 3 & 2 & 5 \\
\hline & Phytodinoacea & Hypnodinium sphaericum & 0 & 1 & 1 \\
\hline Cercizoa & Spongomonadidae & Rhipidodendron sp. & 3 & 0 & 3 \\
\hline Total & & & 350 & 150 & 500 \\
\hline
\end{tabular}


FOLIA Diversidad planctónica de los cuerpos de agua en la Reserva Nacional

Amazónica Allpahuayo Mishana, Loreto - Perú

Tabla 6. Índices de diversidad de fitoplancton y zooplancton en la cuenca del río Nanay en la Reserva Nacional Allpahuayo Mishana (Loreto, Perú).

\begin{tabular}{|c|c|c|c|c|c|c|c|c|c|c|c|c|c|c|c|c|c|c|c|c|c|c|}
\hline \multirow[t]{2}{*}{ Comunidad } & \multirow[t]{2}{*}{ Índices } & \multirow[t]{2}{*}{ Época } & \multicolumn{19}{|c|}{ Estaciones } & \multirow[t]{2}{*}{ Total } \\
\hline & & & Pisco & Anguilla & Yarana & S. Martín & Llanchama & Pintuyacu & $\bar{x}$ & Curaca & Tocón & $\begin{array}{c}\text { S. } \\
\text { Pedro }\end{array}$ & $\begin{array}{c}\text { S. } \\
\text { Cruz }\end{array}$ & $\begin{array}{c}\text { A. } \\
\text { Negra }\end{array}$ & X & Samito & Lagunas & Yuto & Yarana & S.Poza & X & \\
\hline \multirow{12}{*}{ Fitoplancton } & \multirow{2}{*}{ Taxa_S } & $\mathrm{EH}$ & 2.0 & 6.0 & 2.0 & 5.0 & 5.0 & 5.0 & 4.2 & 7.0 & 6.0 & 10.0 & 5.0 & 4.0 & 6.4 & 6.0 & 2.0 & 4.0 & 5.0 & 1.0 & 3.6 & \\
\hline & & ES & 13.0 & 9.0 & 10.0 & 6.0 & 12.0 & 9.0 & 9.8 & 6.0 & 8.0 & 9.0 & 8.0 & 11.0 & 8.4 & 6.0 & 6.0 & 6.0 & 6.0 & 11.0 & 7.0 & \\
\hline & \multirow{2}{*}{ Individuals } & $\mathrm{EH}$ & 4.0 & 10.0 & 5.0 & 7.0 & 12.0 & 8.0 & 7.7 & 10.0 & 13.0 & 10.0 & 10.0 & 15.0 & 11.6 & 16.0 & 8.0 & 7.0 & 12.0 & 3.0 & 9.2 & 150.0 \\
\hline & & ES & 29.0 & 27.0 & 25.0 & 13.0 & 49.0 & 16.0 & 26.5 & 8.0 & 9.0 & 22.0 & 33.0 & 19.0 & 18.2 & 11.0 & 15.0 & 21.0 & 23.0 & 30.0 & 20.0 & 350.0 \\
\hline & \multirow{2}{*}{ Dominance_D } & $\mathrm{EH}$ & 0.6 & 0.2 & 0.5 & 0.3 & 0.4 & 0.3 & 0.4 & 0.2 & 0.2 & 0.1 & 0.2 & 0.3 & 0.2 & 0.2 & 0.5 & 0.3 & 0.2 & 1.0 & 0.5 & \\
\hline & & ES & 0.1 & 0.2 & 0.1 & 0.2 & 0.1 & 0.2 & 0.2 & 0.2 & 0.1 & 0.2 & 0.2 & 0.1 & 0.2 & 0.2 & 0.2 & 0.3 & 0.2 & 0.2 & 0.2 & \\
\hline & \multirow{2}{*}{ Shannon_H } & $\mathrm{EH}$ & 0.6 & 1.6 & 0.7 & 1.5 & 1.2 & 1.4 & 1.2 & 1.8 & 1.7 & 2.3 & 1.5 & 1.3 & 1.7 & 1.6 & 0.7 & 1.4 & 1.5 & 0.0 & 1.0 & \\
\hline & & ES & 2.3 & 1.9 & 2.2 & 1.7 & 2.2 & 2.0 & 2.0 & 1.7 & 2.0 & 2.0 & 1.8 & 2.3 & 2.0 & 1.6 & 1.7 & 1.5 & 1.5 & 2.1 & 1.7 & \\
\hline & \multirow{2}{*}{ Margalef } & $\mathrm{EH}$ & 0.7 & 2.2 & 0.6 & 2.1 & 1.6 & 1.9 & 1.5 & 2.6 & 1.9 & 3.9 & 1.7 & 1.1 & 2.3 & 1.8 & 0.5 & 1.5 & 1.6 & 0.0 & 1.1 & \\
\hline & & ES & 3.6 & 2.4 & 2.8 & 1.9 & 2.8 & 2.9 & 2.7 & 2.4 & 3.2 & 2.6 & 2.0 & 3.4 & 2.7 & 2.1 & 1.8 & 1.6 & 1.6 & 2.9 & 2.0 & \\
\hline & \multirow{2}{*}{ Equitability_J } & $\mathrm{EH}$ & 0.8 & 0.9 & 1.0 & 0.9 & 0.8 & 0.9 & 0.9 & 0.9 & 0.9 & 1.0 & 0.9 & 0.9 & 0.9 & 0.9 & 1.0 & 1.0 & 0.9 & 0.0 & 0.8 & \\
\hline & & ES & 0.9 & 0.9 & 1.0 & 0.9 & 0.9 & 0.9 & 0.9 & 1.0 & 1.0 & 0.9 & 0.9 & 1.0 & 0.9 & 0.9 & 1.0 & 0.8 & 0.9 & 0.9 & 0.9 & \\
\hline \multirow{12}{*}{ Zooplancton } & \multirow{2}{*}{ Taxa_S } & $\mathrm{EH}$ & 2.0 & 6.0 & 3.0 & 6.0 & 7.0 & 6.0 & 5.0 & 4.0 & 5.0 & 2.0 & 3.0 & 5.0 & 3.8 & 4.0 & 3.0 & 6.0 & 5.0 & 7.0 & 5.0 & \\
\hline & & ES & 2.0 & 3.0 & 6.0 & 2.0 & 7.0 & 2.0 & 3.7 & 1.0 & 4.0 & 6.0 & 6.0 & 4.0 & 4.2 & 3.0 & 3.0 & 3.0 & 11.0 & 4.0 & 4.8 & \\
\hline & \multirow{2}{*}{ Individuals } & $\mathrm{EH}$ & 2.0 & 8.0 & 5.0 & 6.0 & 8.0 & 15.0 & 7.3 & 5.0 & 8.0 & 2.0 & 5.0 & 9.0 & 5.8 & 5.0 & 4.0 & 15.0 & 10.0 & 8.0 & 8.4 & 115.0 \\
\hline & & ES & 2.0 & 4.0 & 8.0 & 4.0 & 8.0 & 3.0 & 4.8 & 1.0 & 8.0 & 21.0 & 21.0 & 11.0 & 12.4 & 13.0 & 22.0 & 5.0 & 24.0 & 4.0 & 13.6 & 159.0 \\
\hline & \multirow{2}{*}{ Dominance_D } & $\mathrm{EH}$ & 0.5 & 0.2 & 0.4 & 0.2 & 0.2 & 0.2 & 0.3 & 0.3 & 0.3 & 0.5 & 0.4 & 0.2 & 0.3 & 0.3 & 0.4 & 0.2 & 0.3 & 0.2 & 0.3 & \\
\hline & & ES & 0.5 & 0.4 & 0.2 & 0.6 & 0.2 & 0.6 & 0.4 & 1.0 & 0.3 & 0.4 & 0.4 & 0.3 & 0.5 & 0.5 & 0.5 & 0.4 & 0.1 & 0.3 & 0.3 & \\
\hline & \multirow{2}{*}{ Shannon_H } & $\mathrm{EH}$ & 0.7 & 1.7 & 1.1 & 1.8 & 1.9 & 1.7 & 1.5 & 1.3 & 1.5 & 0.7 & 1.1 & 1.5 & 1.2 & 1.3 & 1.0 & 1.7 & 1.4 & 1.9 & 1.5 & \\
\hline & & ES & 0.7 & 1.0 & 1.7 & 0.6 & 1.9 & 0.6 & 1.1 & 0.0 & 1.2 & 1.3 & 1.3 & 1.2 & 1.0 & 0.9 & 0.8 & 1.0 & 2.3 & 1.4 & 1.3 & \\
\hline & \multirow{2}{*}{ Margalef } & $\mathrm{EH}$ & 1.4 & 2.4 & 1.2 & 2.8 & 2.9 & 1.8 & 2.1 & 1.9 & 1.9 & 1.4 & 1.2 & 1.8 & 1.7 & 1.9 & 1.4 & 1.8 & 1.7 & 2.9 & 2.0 & \\
\hline & & ES & 1.4 & 1.4 & 2.4 & 0.7 & 2.9 & 0.9 & 1.6 & 0.0 & 1.4 & 1.6 & 1.6 & 1.3 & 1.2 & 0.8 & 0.6 & 1.2 & 3.1 & 2.2 & 1.6 & \\
\hline & \multirow{2}{*}{ Equitability_J } & $\mathrm{EH}$ & 1.0 & 1.0 & 1.0 & 1.0 & 1.0 & 0.9 & 1.0 & 1.0 & 0.9 & 1.0 & 1.0 & 0.9 & 1.0 & 1.0 & 0.9 & 0.9 & 0.9 & 1.0 & 0.9 & \\
\hline & & ES & 1.0 & 0.9 & 1.0 & 0.8 & 1.0 & 0.9 & 0.9 & 0.0 & 0.9 & 0.7 & 0.7 & 0.9 & 0.6 & 0.8 & 0.7 & 0.9 & 0.9 & 1.0 & 0.9 & \\
\hline
\end{tabular}

Nota: ES Época Seca y EH Época Húmeda

Fuente: Tesis, Carlos Chávez, 2019 
Tabla 7. Abundancia del zooplancton en la cuenca del río Nanay, Reserva Nacional Allpahuayo Mishana (Loreto, Perú).

\begin{tabular}{|c|c|c|c|c|c|c|}
\hline & taciones & & & Total Seca & $\begin{array}{c}\text { Total } \\
\text { Húmeda }\end{array}$ & TOTAL \\
\hline Phylum & Clase & Familia & Especie & & & \\
\hline \multirow{6}{*}{ Protozoa } & \multirow{6}{*}{ Rhizopoda } & \multirow{4}{*}{ Arcellidae } & Arcella vulgaris & 36 & 17 & 53 \\
\hline & & & Arcella discoides & 0 & 4 & 4 \\
\hline & & & Arcella dentata & 6 & 12 & 18 \\
\hline & & & Euglypha sp. & 3 & 5 & 8 \\
\hline & & \multirow{2}{*}{ Difflugiidae } & Difflugia sp. & 1 & 5 & 6 \\
\hline & & & Centropyxes sp. & 3 & 2 & 5 \\
\hline \multirow{18}{*}{ Rotatoria } & \multirow{18}{*}{ Monogononta } & \multirow{12}{*}{ Brachionidae } & Brachionus sp. & 10 & 15 & 25 \\
\hline & & & Brachionus falcatus & 1 & 0 & 1 \\
\hline & & & Brachionus gessneri & 12 & 0 & 12 \\
\hline & & & Brachionus calyciflorus & 2 & 0 & 2 \\
\hline & & & Brachionus zhanisseri & 1 & 0 & 1 \\
\hline & & & Monostyla sp. & 4 & 5 & 9 \\
\hline & & & Notholca striata & 0 & 8 & 8 \\
\hline & & & Lecane sp. & 13 & 3 & 16 \\
\hline & & & Lepadella sp. & 2 & 0 & 2 \\
\hline & & & Keratella sp. & 5 & 0 & 5 \\
\hline & & & Anuraeopsis sp. & 3 & 0 & 3 \\
\hline & & & Mytillina sp. & 1 & 0 & 1 \\
\hline & & \multirow{2}{*}{ Asplanchnidae } & Asplanchnopus sp. & 3 & 0 & 3 \\
\hline & & & Asplanchna sp. & 4 & 0 & 4 \\
\hline & & Pleosomatidae & Pleosoma sp. & 1 & 0 & 1 \\
\hline & & Synchaetidae & Polyarthra trigla & 6 & 3 & 9 \\
\hline & & Trichocercidae & Trichocerca sp. & 6 & 11 & 17 \\
\hline & & Testudinellidae & Filinia longiseta & 12 & 5 & 17 \\
\hline \multirow{4}{*}{ Arthropoda } & Copepoda & Cyclopoidae & Indeterminado & 3 & 4 & 7 \\
\hline & \multirow{3}{*}{ Cladocera } & Bosminidae & Bosminopsis sp. & 21 & 4 & 25 \\
\hline & & Chydoridae & Pleuroxus sp. & 0 & 2 & 2 \\
\hline & & Chironomidae & Chironomus sp. & 0 & 10 & 10 \\
\hline Total & & & & 159 & 115 & 274 \\
\hline
\end{tabular}


El 56,25\% de las estaciones (3 en río, 3 en quebradas y 3 en cochas) presentaron relativamente mayor riqueza de especies en la época húmeda, el 25\% de estaciones (1 en río, 2 en quebradas y 1 en cocha) presentaron mayor riqueza en la época seca comparada con la época húmeda, y el 18,75\% (2 estaciones de río y 1 de cocha) presentaron la misma riqueza especifica en ambas épocas (Tabla 4).

A nivel de medias según el tipo de cuerpo de agua, en época seca hubo mayor riqueza en las cochas, seguido de las quebradas y luego por las estaciones en ríos; mientras que, en la época húmeda, tanto las cochas como las estaciones en río tuvieron similar riqueza de especies, dejando en último lugar a las quebradas.

Comparando las dos épocas de monitoreo y el tipo de cuerpo de agua, la cocha Yarana presentó mayor riqueza especifica con 11 especies en la época seca, seguida de la cocha Supay Poza con 7 especies en época húmeda, y cocha Llanchama con 7 especies en ambas épocas. La estación con menos riqueza absoluta fue la cocha Curaca que tuvo una sola especie del phylum Protozoa en la época seca.

En época seca se registraron 159 individuos, mientras que, en época húmeda se registraron 115 individuos, haciendo un total de 274. El phylum Rotatoria fue el más abundante $(49,6 \%)$, seguido de Protozoa (34,3\%), y, por último, Arthropoda (16,1\%), ver Tabla 7.

Los índices de diversidad para zooplancton se aprecian en la Tabla 6. Los valores más altos de diversidad de Shannon-Weaver se registraron en las cochas Yarana y LLanchama, ambos en temporada seca, mientras que en temporada húmeda nuevamente LLanchama y Supay Poza tuvieron los valores más altos, considerados como de diversidad normal.

Por otro lado, en la temporada seca, la media con mayor diversidad entre todos los cuerpos de agua estuvo en las cochas $(\mathrm{H}=1,27$ bits), seguida de las estaciones ubicadas en los ríos $(\mathrm{H}=1,10$ bits), $y$, por último, el de las quebradas $(\mathrm{H}=1,02$ bits). Entretanto, en la temporada húmeda, las estaciones en los ríos mostraron la media más alta $\mathrm{H}=1,48$ bits, seguido por las cochas $(\mathrm{H}=1,47$ bits) y por último las quebradas ( $\mathrm{H}=1,22$ bits).

El índice de Margalef más alto se registró en cocha Yarana $(d=3,15)$, seguido de la cocha LLanchama $(d=2,89)$, ambas en la época seca. Mientras que LLanchama y la cocha Supay Poza $(d=2,89)$ fueron las estaciones con mayor índice en la época húmeda. A nivel de medias entre los cuerpos de agua, en época seca destacan las estaciones en los ríos, seguido de las cochas y, por último, las quebradas. Mientras que en la temporada húmeda el orden de las medias fue mayor en las estaciones en ríos, seguido de las cochas y finalmente en las quebradas.

En la estación seca el mayor índice de Simpson se registró en la quebrada Curaca $(D=1,00)$, cuyo valor nos indica que existe dominio de una especie en particular, es decir, existe una alta dominancia. El orden por tipo de cuerpo de agua fue quebradas, ríos y cochas. De otro lado, en la época húmeda Pisco y quebrada San Pedro presentaron índices de 0,50, seguido de la cocha Lagunas $(D=0,38)$, luego la estación en río Yarana y quebrada Santa Cruz, (ambos con $\mathrm{D}=0.36$ ). El orden según las medias fue quebradas, ríos y cochas.

En la época seca los índices de equidad fueron superiores a $\mathrm{J}=0,73$, presentando una equidad relativamente alta y una distribución relativamente homogénea. Las estaciones con la más alta equidad $(\mathrm{J}=1,00)$ fueron Pisco y cocha Supay Poza; seguidos de las estaciones en río LLanchama y Yarana. A nivel de medias por tipo de cuerpos de agua el orden fue estaciones en río, cochas y quebradas. En época húmeda, los valores obtenidos fueron superiores a 0,88. Las estaciones con índice $(\mathrm{J}=1,00)$ fueron Pisco, San Martín y la quebrada San Pedro. El orden según 
medias por tipo de cuerpo de agua fue ríos, quebradas y cochas.

\section{DISCUSIÓN}

Bacillariophyta representó aproximadamente el $60 \%$ del fitoplancton en ambas épocas de estudio, coincidiendo con lo reportado por López $\&$ Vela (2011), quienes indican que las diatomeas (Bacillariophyta) presentaron la mayor densidad de organismos durante su estudio en la cocha Llanchama, ambiente de aguas negras ubicada en la cuenca del río Nanay, demostrando que la cuenca aún mantiene su estado de conservación y coincidiendo con Lobo et al. (2004), Ortega et al. (2010), y con López \& Siqueiros (2011), quienes indican que las diatomeas son buenas indicadoras de calidad de agua y que su diversidad se corresponde con la agradable apariencia y estado prístino de los mismos, ya que este recurso es la fuente para el servicio de agua potable de la ciudad de Iquitos.

Bacillariophyta fue el grupo predominante en este estudio, resultado similar a lo reportado por Ismiño et al. (2018), en la cuenca del río Itaya, que también presenta características físicas y químicas típicas de un río de agua negra (Maco, 2006).

Para el caso del zooplancton en la época seca la mayor riqueza de especies, así como la mayor abundancia de individuos fueron encontradas en las cochas, corroborando lo descrito por Pinto \& Santos-Silva (2018) quienes indican que en la época seca generalmente se encuentra mayor densidad poblacional debido a la gran cantidad de material en suspensión de dichos ambientes y donde quedan más concentrados.

El 50\% aproximadamente del zooplancton en este ecosistema de agua negra estuvo dominado por el phylum Rotatoria, por encima de los protozoarios, cladóceros y copépodos, en las dos épocas de estudio. Estos resultados coinciden con lo reportado por Riofrio et al. (2003), quienes realizaron la caracterización limnológica de la laguna Cashibococha (Ucayali, Perú), cuyas características físicas y químicas la identifican como un cuerpo de agua negra, con $\mathrm{pH}$ ligeramente ácido, baja alcalinidad, baja conductividad y moderada transparencia, donde el phylum Rotatoria abarcó aproximadamente el $80 \%$ del zooplancton. Asimismo, nuestros resultados coinciden con el trabajo realizado por Calixto et al. (2011) en el lago Tupé, un lago de tiene influencia directa de quebradas no inundables y del río Negro, donde Rotífera presentó mayor riqueza de especies y densidad poblacional, seguido por Cladocera y Copepoda.

Con relación al índice de Shannon-Weaver para fitoplancton y zooplancton, en época seca los valores registrados fueron mayores a los obtenidos en la época húmeda, debido a una mayor dispersión de los especímenes por consecuencia al mayor volumen de agua presente en el ecosistema, corroborando lo descrito por Pinto \& Santos-Silva (2018) e indicando que la cuenca media del río Nanay presenta grupos taxonómicos de biodiversidad media o normal, similar a lo encontrado por Ismiño et al. (2018) en la cuenca del río Itaya.

Con relación a los índices de Margaleff para fitoplancton y zooplancton, en ambas épocas de estudio, demostraron que dichos valores de diversidad estuvieron relacionados con la riqueza y la abundancia que presentaron los diferentes ambientes, siendo similares a los registrados por Walsh Perú (2014).

Con relación al índice de Simpson, mientras que en la época seca se registraron en algunas estaciones los máximos valores para fitoplancton, a su vez se registraron en algunas estaciones los menores valores para el zooplancton; sucediendo lo contrario en la época húmeda. Sin embargo, a nivel global no existe dominio de una especie en particular en toda la cuenca estudiada, 
reportándose una baja dominancia, lo cual coincide con lo reportado por Walsh Perú (2014) quienes estudiaron diversos ríos y quebradas en ambas épocas en la selva central del Perú.

Los valores encontrados para equidad en fitoplancton y zooplancton mostraron índices de Pielou similares a los indicados por Ferreira y Jegú en Riofrio et al. (2003) y por Ismiño et al. (2018) quienes consideran que dichos índices son indicadores de comunidades en equilibrio y cuyas poblaciones son homogéneas.

\section{AGRADECIMIENTO}

Al personal de la ONG Centro de Investigación y Promoción Popular - CENDIPP, a la Universidad Nacional de la Amazonía Peruana - UNAP, al Servicio Nacional de Áreas Naturales Protegidas - SERNANP, al Instituto de Investigaciones de la Amazonía Peruana - IIAP, quienes apoyaron al desarrollo del estudio.

\section{REFERENCIAS BIBLIOGRÁFICAS}

Aldave, A. 1989. Algas. Editorial Libertad, Trujillo, Perú. 269 pp.

Bicudo, C.E.; Bicudo, T. 1969. Algas de aguas continentais brasileras. Fundaçao Brasileira para o Desenvolvimento do Ensino de Ciências, São Paulo, Brasil. 277 pp.

Bicudo, C.E.; Menezes, M. 2006. Géneros de algas continentais do Brasil. RiMa Editora, São Carlos, Brasil. 489 pp.

Bourrely, P. 1968. Les algues d'eau douce. Tomo II Les algues jaunes et brunes Chrysophycees, Pheophycees, Xanthophycees et Diatomees. Editions N. Boubee \& Cie, Paris. 438 pp.

Bourrely, P. 1970. Les algues d'eau douce. Tomo III Les algues bleues et rouges les Eugleniens, Peridiniens et Crytomonadines. Editions N. Boubee \& Cie, Paris. 450 pp.
Bourrely, P. 1972. Les algues d'eau douce. Tomo I Les algues vertes. Editions N. Boubee \& Cie, Paris. 470 pp.

Cadima, M.F; Bicudo, C.E. 2014. Guía ilustrada de algas de Bolivia división Euglenophyta. $2^{a}$ ed. Universidad Mayor de San Simón, Cochabamba. 263 pp.

Calixto, L.S.E.F.; Ghidini, A.R.; Silva, E.A.; SantosSilva, E.N. 2011. Distribuição espaço-temporal do zooplâncton no lago Tupé, baixo rio Negro, Amazonas, Brasil. En: Santos-Silva, E.N.; Scudeller, V.V.; Cavalcanti, M.J. (Eds). BioTupé: meio físico, diversidade biológica e sociocultural do Baixo Rio Negro, Amazônia Central - v. 03. Manaus, p. 201-230.

Cambra, J.; Ector, L; Sabater, S. 2005. Protocolos de muestreo y análisis para fitobentos. In: De la Fuente, M. (Ed). Metodología para el establecimiento del estado ecológico según la directiva marco del agua en la Confederación Hidrográfica del Ebro. Ministerio del Medio Ambiente. p. 64-95.

Eddy, S.; Hodson, A.C. 1961. Taxonomic keys. 3th ed. Burguess, Minnesota. 162 pp.

Fernández, H.A. 1982. Guía para el estudio de las algas. Tall. Graf. I.V.P., Trujillo, Perú. 263 pp.

Goswami, S.C. 2004. Zooplankton methodology, collection and identification: a field manual. National Institute of Oceanography, Goa, India. $26 \mathrm{pp}$.

Instituto de Investigaciones de la Amazonía Peruana - IIAP/BIODAMAZ, 2004. Bases biofísicas y propuestas de zonificación y de programas para el plan maestro de la Zona Reservada Allpahuayo Mishana, IIAP, Iquitos, $87 \mathrm{pp}$.

Instituto de Investigaciones de la Amazonía Peruana - IIAP, 2009. Viabilidad económica de la pesca artesanal en el departamento de Loreto, IIAP, Iquitos, $47 \mathrm{pp}$.

Ismiño, R.; Montalván, G.; García, A; Maco, J.; Tello, S.; Palacios, J.J.; Rodríguez, L. 2018. Comunidad 
fitoplanctónica de la cuenca del río Itaya en Loreto, Perú. Revista de Investigaciones Veterinarias del Perú, 29(3): 828-839. DOI: https://doi.org/10.15381/rivep.v29i3.14766

Lenz, J. 2000. Introduction. In: Harris, R.P.; Wiebe, P.H.; Lenz, J.; Skjoldal, H.R.; Huntley, M. (Eds.). ICES zooplankton metodology manual. p. 1-32. Lobo, E.A.; Callegaro, V.L.M.; Hermany, G.; Bes, D.; Wetzel, C.A.; Oliveira, M.A. 2004. Use of epilithic diatoms as bioindicators from lotic systems in southern Brazil, with special emphasis on eutrophication. Acta Limnologica Brasiliensia, 16(1): 25-40.

López F.F.O.; Siqueiros B.D.A. 2011. Las Diatomeas como indicadores de la calidad ecológica de los oasis de Baja California Sur, México. Biodiversitas, 99: 8-11.

López, S.R.; Vela, G.L. 2011. Densidad y diversidad del fitoplancton de la cocha Llanchama, en los periodos hidrológicos de creciente $\mathrm{y}$ media vaciante, Loreto, Perú. Conocimiento Amazónico, 2(2): 157-166.

Maco, GJ. 2006. Tipos de ambientes acuáticos de la Amazonía Peruana. Folia Amazónica, 15(12): 131-140. DOI: https://doi.org/10.24841/ fa.v15i1-2.231

Ministerio del Ambiente - MINAM; Universidad Nacional Mayor de San Marcos - UNMSM, 2014. Manual de métodos de colecta, identificación y análisis de comunidades biológicas: plancton, perifiton, bentos (macroinvertebrados) $y$ necton (peces) en aguas continentales del Perú, MINAM, Lima. 77 pp.

Ortega, H.; Chocano, E.; Palma, C.; Samanez, I. 2010. Biota acuática en la Amazonía Peruana: diversidad y usos como indicadores ambientales en el Bajo Urubamba (CuscoUcayali). Revista Peruana de Biología, 17(1): 29-35. DOI: https://doi.org/10.15381/rpb. v17i1.47
Pennak, R.W. 1978. Fresh-water invertebrates of the United States. $2^{\text {nd }}$ ed. John Wiley \& Sons, New York. 803 pp.

Pinto, F.S.W.O.; Santos-Silva, E.N. 2018. Riqueza, composição, abundância e distribuição de ciclopóides nas regiões litorânea e limnética do lago Tupé, Manaus, AM, Brasil. Scientia Amazonia, 7(1): 97-106.

Ríos, E.; Sánchez, H.; Cubas, R.; Montreuil, V.; Alcántara, F.; Del Águila, M.; Pizango, E. 2002. Migración pasiva del ictioplancton en la cuenca media del río Nanay - Loreto - Perú: informe final de proyecto. Universidad Nacional de la Amazonía Peruana, Iquitos. 45 pp.

Riofrio, J.; Samanez, I.; Carrasco, F.; Clavo, M. 2003. Caracterización limnológica de la laguna de Cashibococha (Ucayali, Perú) durante el año 2001. Revista Peruana de Biología, 10(2): 183-194. DOI: https://doi.org/10.15381/rpb. v10i2.2501

Servicio Nacional de Áreas Protegidas por el Estado - SERNANP, 2013. Plan maestro de la Reserva Nacional Allpahuayo Mishana 20132018. SERNANP, Iquitos. $24 \mathrm{pp}$.

Sipaúba-Tavares, L.H.; Rocha, O. 2001. Produção de plâncton (fitoplâncton e zooplâncton) para alimentação de organismos aquáticos. RiMa editora, São Carlos. 106 pp.

Walsh Perú 2014. Línea base ambiental del área de influencia del proyecto. In: Walsh Perú (Ed). Estudio de Impacto Ambiental para la perforación de pozos exploratorios desde 10 Plataformas en el Lote 108. p. 73-694.

Wittmann, F.; Junk, W.J. 2016. Amazon river basin. In: Finlayson, C.; Milton, G.; Prentice, R.; Davidson, N. (Eds). The wetland book 2: distribution, description and conservation. Springer, Amsterdam. p. 1-16.

Recibido: 2 de febrero de 2020 Aceptado para publicación: 21 de diciembre de 2020

Esta obra está bajo una Licencia Creative Commons Atribución-NoComercial-SinDerivar 4.0 Internacional.

Vol. 29 (2) 2020. 353-370

DOI: https://doi.org/10.24841/fa.v29i2.536 